\title{
Expression profiling of familial breast cancers demonstrates higher expression of FGFR2 in BRCA2-associated tumors
}

\author{
Anita L. Bane · Dushanthi Pinnaduwage • \\ Sarah Colby · Shelley B. Bull · Frances P. O'Malley • \\ Irene L. Andrulis
}

Received: 21 February 2008/Accepted: 30 May 2008/Published online: 18 June 2008

(c) The Author(s) 2008

\begin{abstract}
Background BRCA1- and BRCA2-associated tumors appear to have distinct molecular signatures. $B R C A 1$-associated tumors are predominantly basal-like cancers, whereas BRCA2-associated tumors have a predominant luminal-like phenotype. These two molecular signatures reflect in part the two cell types found in the terminal duct lobular unit of the breast. To elucidate novel genes involved in these two spectra of breast tumorigenesis we performed global gene expression analysis on breast
\end{abstract}

Electronic supplementary material The online version of this article (doi:10.1007/s10549-008-0087-1) contains supplementary material, which is available to authorized users.

A. L. Bane · F. P. O’Malley · I. L. Andrulis

Department of Pathology and Laboratory Medicine, Mount Sinai Hospital, 600 University Ave., Toronto, ON, Canada M5G 1X5

A. L. Bane · I. L. Andrulis ( $\square)$

Fred A. Litwin Centre for Cancer Genetics, \#984 Samuel

Lunenfeld Research Institute, Mount Sinai Hospital,

600 University Ave., Toronto, ON, Canada M5G 1X5

e-mail: andrulis@mshri.on.ca

A. L. Bane · F. P. O’Malley · I. L. Andrulis

Department of Pathology and Laboratory Medicine,

University of Toronto, Toronto, ON, Canada

D. Pinnaduwage $\cdot$ S. Colby $\cdot$ S. B. Bull

Prosserman Center for Health Research, Samuel Lunenfeld

Research Institute, Mount Sinai Hospital, 600 University Ave.,

Toronto, ON, Canada M5G 1X5

S. B. Bull

Department of Public Health Sciences, University of Toronto, Toronto, ON, Canada

I. L. Andrulis

Department of Molecular and Medical Genetics,

University of Toronto, Toronto, ON, Canada tumors from germline $B R C A 1$ and $B R C A 2$ mutation carriers. Methodology Breast tumor RNAs from $7 B R C A 1$ and 6 BRCA2 mutation carriers were profiled using UHN human 19K cDNA microarrays. Supervised univariate analyses were conducted to identify genes differentially expressed between BRCA1 and BRCA2-associated tumors. Selected discriminatory genes were validated using real time reverse transcription polymerase chain reaction in the tumor RNAs, and/or by immunohistochemistry (IHC) or by in situ hybridization (ISH) on tissue microarrays (TMAs) containing an independent set of 58 BRCA1 and 64 $B R C A 2$-associated tumors. Results Genes more highly expressed in BRCAl-associated tumors included stathmin, osteopontin, TGF $\beta 2$ and Jagged 1 in addition to genes previously identified as characteristic of basal-like breast cancers. BRCA2-associated cancers were characterized by the higher relative expression of $F G F 1$ and $F G F R 2$. FGFR2 protein was also more highly expressed in BRCA2associated cancers $(P=0.004)$. Significance BRCAlassociated tumours demonstrated increased expression of component genes of the Notch and TGF $\beta$ pathways whereas the higher expression of FGFR2 and FGF1 in $B R C A 2$-associated cancers suggests the existence of an autocrine stimulatory loop.

Keywords $B R C A 1 \cdot B R C A 2 \cdot$ Expression profiling · Familial breast cancer

\section{Introduction}

$B R C A 1$ - and BRCA2-associated tumors have many morphologic features in common. These include ductal histology, high histologic grade, pushing tumor margins and a notable host lymphocytic response [1, 2]. Despite 
these similarities they have distinct molecular signatures; $B R C A 1$-associated tumors have been shown to be predominantly estrogen receptor negative and to have a basal phenotype [3-5], whereas we have shown previously that BRCA2-associated tumors have a luminal phenotype characterized by the expression of estrogen receptor and luminal-type cytokeratins [1]. These two molecular signatures reflect in part the two cell types, basal/myoepithelial and luminal found in the terminal duct lobular unit of the normal breast.

Basal-like human breast cancers have been shown to exhibit aberrations in the epidermal growth factor receptor (EGFR) signaling pathway [6]. With the exception of Cyclin D1, genes responsible for promoting the growth and survival of BRCA2-associated cancers have yet to be identified [7]. Our objective in performing global gene expression analysis on BRCA1- and BRCA2-associated tumors was to distinguish these distinct pathways of carcinogenesis and to elucidate novel genes necessary for the transformation and survival of BRCA2-associated tumors.

\section{Materials and methods}

\section{Breast cancer cases}

Flash frozen tumor tissue was available from 7 germline BRCA1 carriers and 6 germline BRCA2 carriers participating in the Ontario Familial Breast Cancer Registry (OFBCR) $[8,9]$ and the Ontario Cancer Genetics Network (OCGN). Following pathologic confirmation of invasive disease, the tumor tissue was frozen and stored in liquid nitrogen. Testing for germline mutations in BRCAl and $B R C A 2$ was performed using an RNA/DNA-based protein truncation test with complementary $5^{\prime}$ sequencing as previously described [10]. All mutations were confirmed by DNA sequencing. Mutations were classified as deleterious if they were protein-truncating, missense mutations (rare), or splice-site mutations as defined by the Breast Informatics Consortium (http://research.nhgri.nih.gov/bic/).

The clinicopathologic characteristics and mutation status (for the BRCA1-associated and BRCA2-associated tumors) are summarized in Table 1. Tumors were classified according to the WHO histologic classification of breast tumors [11] and graded by central pathology review using the Nottingham histologic grading system [12]. The pathologist was blinded to the mutational status or family history of the participants. Receptor status was obtained by either biochemical or immunohistochemical methods.

A validation set of formalin-fixed paraffin-embedded (FFPE) tumors from $58 \mathrm{BRCA1}$ carriers, $64 \mathrm{BRCA2}$ carriers and 242 additional individuals from the OFBCR without $B R C A 1$ or $B R C A 2$ mutations was used to construct tissue microarrays (TMAs).

RNA extraction and reverse transcription

Total RNA was isolated from 20 to $50 \mathrm{mg}$ of tumor tissue using TRIzol reagent (Gibco BRL Life Technologies). Reference RNA was a pool of 13 cell lines, modified from the common reference cell line list [13]. Tumor and reference RNAs $(5 \mu \mathrm{g})$ were reverse transcribed with
Table 1 Clinicopathologic characteristics of $B R C A 1$ - and $B R C A 2$-associated breast cancers

\footnotetext{
${ }^{\text {a }}$ ID: Identification number

${ }^{b}$ ER: Estrogen receptor

c PR: Progesterone receptor

d NST: No special type

e Neg: Negative

${ }^{\mathrm{f}}$ Equ: Equivalent

g Pos: Positive

h NA: Not available
}

\begin{tabular}{|c|c|c|c|c|c|c|c|c|}
\hline $\begin{array}{l}\mathrm{LAB} \\
\mathrm{ID}^{\mathrm{a}}\end{array}$ & Mutation & $\begin{array}{l}\text { Type of } \\
\text { invasive } \\
\text { cancer }\end{array}$ & $\begin{array}{l}\text { Age at } \\
\text { diagnosis }\end{array}$ & Grade & $\begin{array}{l}\text { Lymphatic } \\
\text { invasion }\end{array}$ & $\begin{array}{l}\text { Blood } \\
\text { vessel } \\
\text { invasion }\end{array}$ & $\mathrm{ER}^{\mathrm{b}}$ & $\mathrm{PR}^{\mathrm{c}}$ \\
\hline
\end{tabular}

\begin{tabular}{|c|c|c|c|c|c|c|c|c|}
\hline \multicolumn{9}{|c|}{ BRCA1 mutation } \\
\hline 3435 & $2457 \mathrm{C}-\mathrm{T}$ & Ductal, NST ${ }^{\mathrm{d}}$ & 50 & 3 & Absent & Absent & $\mathrm{Neg}^{\mathrm{e}}$ & Neg \\
\hline 4080 & 185delAG & Ductal, NST & 40 & 3 & Absent & Absent & $\mathrm{Equ}^{\mathrm{f}}$ & $\operatorname{Pos}^{\mathrm{g}}$ \\
\hline 4326 & $185 \mathrm{delAG}$ & Ductal, NST & 35 & 3 & Absent & Absent & $\mathrm{NA}^{\mathrm{h}}$ & $\mathrm{Neg}$ \\
\hline 1834 & 5293delAAAG & Ductal, NST & 30 & 3 & Present & Absent & $\mathrm{Neg}$ & NA \\
\hline 1693 & 5382insC & Ductal, NST & 44 & 3 & Absent & Absent & Equ & Pos \\
\hline 2528 & 4603G-T & Ductal, NST & 33 & 3 & NA & NA & $\mathrm{Neg}$ & Pos \\
\hline 3078 & $185 \mathrm{delAG}$ & Ductal, NST & 32 & 3 & Present & Present & $\mathrm{Neg}$ & Equ \\
\hline \multicolumn{9}{|c|}{$B R C A 2$ mutation } \\
\hline 4324 & 8765delAG & Ductal, NST & 44 & 3 & Present & Absent & $\mathrm{Neg}$ & Pos \\
\hline 4472 & IVS16 + 3 A-C & Ductal, NST & 44 & 3 & Absent & NA & $\mathrm{Neg}$ & $\mathrm{Neg}$ \\
\hline 2172 & 6174delT & Ductal, NST & 46 & 3 & Present & NA & Pos & Pos \\
\hline 3627 & $9132 \mathrm{delC}$ & Ductal, NST & 34 & 3 & Present & Absent & Pos & Pos \\
\hline 4374 & 8765delAG & Ductal, NST & 58 & 3 & Present & Absent & $\mathrm{Neg}$ & Neg \\
\hline 3242 & 6764insA & Ductal, NST & 75 & 3 & Present & Absent & Pos & Equ \\
\hline
\end{tabular}


Superscript III reverse transcriptase (Invitrogen) to yield cDNA. Tumor and reference cDNA $(5 \mu \mathrm{g})$ were indirectly labeled using aminoallyl nucleotide analogs with $\mathrm{Cy} 3$ and Cy5 fluorescent tags, respectively.

Dye labeling and cDNA array hybridisation

The labeled probes were simultaneously hybridized to UHN human $19 \mathrm{~K}$ cDNA microarray slides (www. microarrays.ca), and incubated overnight at $42^{\circ} \mathrm{C}$. These 19K cDNA microarrays are single-spotted and contain 19,008 characterized or unknown human expressed sequence tags (ESTs). The clone set has been sequenceverified at the UHN Microarray Centre, Toronto. Following hybridization the slides were washed and scanned using an Axon scanner. Fluorescent dye swap experiments were also performed for two tumors.

Pre-processing of expression data

The gene expression data were obtained from the original image files as spot intensities by correcting the mean foreground for each spot with the median local background. The array quality was controlled by requiring arrays to have more than $80 \%$ of spots with spot intensities higher than their local background and more than $75 \%$ of spots with spot intensity higher than 1.2 times their local background in both channels [14]. Spots with foreground intensity lower than background were treated as missing. A relative expression value was obtained for each gene as the log base 2 ratio of the adjusted intensity for the sample channel versus the reference channel. The $\log 2$ ratios were normalized by a within-subarray print-tip "loess" adjustment, followed by a between-array scale adjustment [15]. Poor quality spots as flagged by the GenePix image analysis software were excluded from the normalization. Pre-processing and normalization were carried out using the R-Bioconductor package LIMMA [16]; http://www.Bioconductor.org). There were 18,981 genes retained in the final data set for analysis.

\section{TaqMan ${ }^{\circledR}$ assay-based real-time RT-PCR}

mRNA expression levels of six selected genes that were statistically significantly differentially expressed between the two tumor groups and/or of biologic interest were measured in representative tumor specimens by real-time reverse transcription-polymerase chain reaction (RT-PCR) using TaqMan ${ }^{\circledR}$ PRISM 7500 Fast Real-Time PCR System (Applied Biosystems, Foster City, CA). Gene expression levels were determined by quantification relative to a control gene, hypoxanthine guanine phosphorbosyl transferase (HPRT). cDNA was generated using the ABI High Capacity cDNA Archiving Kit (Applied Biosystems, Foster
City, CA) and RT-PCR reactions were carried out following the manufacturer's protocol.

Tissue microarrays

Tissue microarrays were constructed as previously described [1] using FFPE tumors from 58 BRCAl carriers, 64 $B R C A 2$ carriers and 242 individuals without $B R C A 1$ or $B R C A 2$ mutations from the OFBCR. $4 \mu \mathrm{m}$ thick sections of these blocks were used for immunohistochemical staining with the FGFR2 antibody (polyclonal, Abcam). $5 \mu \mathrm{m}$ thick sections were used for mRNA in situ hybridization (ISH). ISH analysis of breast cancer has been reported in detail elsewhere previously [17]. Briefly, a cDNA probe for ISH was made to the gene of interest using 33P-UTP-radiolabeled cRNA. Using routine techniques the TMA sections were hybridized with the radiolabeled antisense probe, washed, and treated with Kodak NBT-2 nuclear emulsion. The sections from the TMAs were stored at $4{ }^{\circ} \mathrm{C}$ for several weeks prior to development in Kodak D-19 solution; they were subsequently fixed in Kodafix and counterstained with $0.1 \%$ toludine blue.

Immunoreactivity and mRNA ISH was scored using the Allred method [18], which combined the intensity of staining with the percentage of positive tumor cells observable resulting in a combined score of $0-8$. For FGFR2, a score of 7 or above was considered positive; for Jagged 1, a previously determined score of 4 or higher [17] was considered positive.

\section{Statistical analysis}

To identify genes that discriminate between $B R C A l$ - and $B R C A 2$-associated tumors, supervised univariate analyses of array-based $\log 2$ gene expression were performed for each clone treating the modified Student $t$-test as the primary analysis [19] using the SAM procedure implemented in open source software, version 2.1.0 (http://www.r-project.org/). To assess the sensitivity of the results to this analysis method the random variance test was also applied using BRB Array Tools (http://linus.nci.nih.gov/BRB-ArrayTools.html). For each test, all clones were ranked according to their ability to discriminate $B R C A 1$-associated tumors from $B R C A 2$-associated tumors. Supplementary Table 1 reports discriminating genes with $P$-values $\leq 0.01$ according to the modified Student $t$-test. $P$-values were not adjusted for multiple testing.

$\log 2$ transformed relative expression of genes validated by RT-PCR was compared to array-based $\log 2$ gene expression via scatter plots. Means of the RT-PCR expressions were compared between the two BRCA tumor groups using the Student's $t$-test and Welch unequal 
variance $t$-tests. All tests were two-sided. $P$-values were not adjusted for multiple testing.

The proportion of tumors positive by immunohistochemical staining and mRNA ISH for BRCAl-associated and $B R C A 2$-associated tumors were compared using Fisher's exact test for association. All tests were two sided. Statistical analysis was performed using SAS 9.1 software (SAS, Institute Inc., Cary, North Carolina). $P$-values were not adjusted for multiple testing.

\section{Results}

Clinicopathologic characteristics of BRCAland $B R C A 2$-associated tumors

Thirteen tumors were obtained from individuals known to harbor a $B R C A 1$ or $B R C A 2$ germline mutation. As indicated in Table 1 , all of the $13 B R C A$-associated tumors were grade III/III invasive ductal, no special type tumors
(NST). Both ER positive/equivalent and negative tumors were included among the $B R C A 1$ and $B R C A 2$ carriers. The mean age at diagnosis of the BRCAl patients was somewhat younger than the mean age for the $B R C A 2$ subjects (38 vs. 43 years).

Identification of genes that distinguish BRCAIand $B R C A 2$-associated breast tumors

The modified Student $t$-test was used to identify clones on the arrays that were differentially expressed between the $B R C A 1$ - and BRCA2-associated breast tumors. There were 4 distinguishing clones with a $P$-value $<0.001$ and 127 clones with a $P$-value $<0.01$. The 150 top-ranked differentially expressed genes with a $P$-value of $\leq 0.01$ are listed in supplementary Table 1 and displayed as a heat map, Fig. 1.

$B R C A 1$-associated tumors were characterized by the higher relative expression of 52 of these clones. Using the gene ontology database (NCBI), these genes was predicted to be involved in diverse cellular functions such as
Fig. 1 Unsupervised two dimensional cluster analysis of 7 BRCA1-associated and 6 $B R C A 2$-associated breast tumors. Two dimensional presentation of transcript ratios for 13 tumors. The top 150 significant genes differentially expressed between the two tumor groups from SAM Moderated $t$-test are shown. Each column represents a tumor and each row a gene. As shown in the color bar, red indicates up regulation, green down regulation and black no change

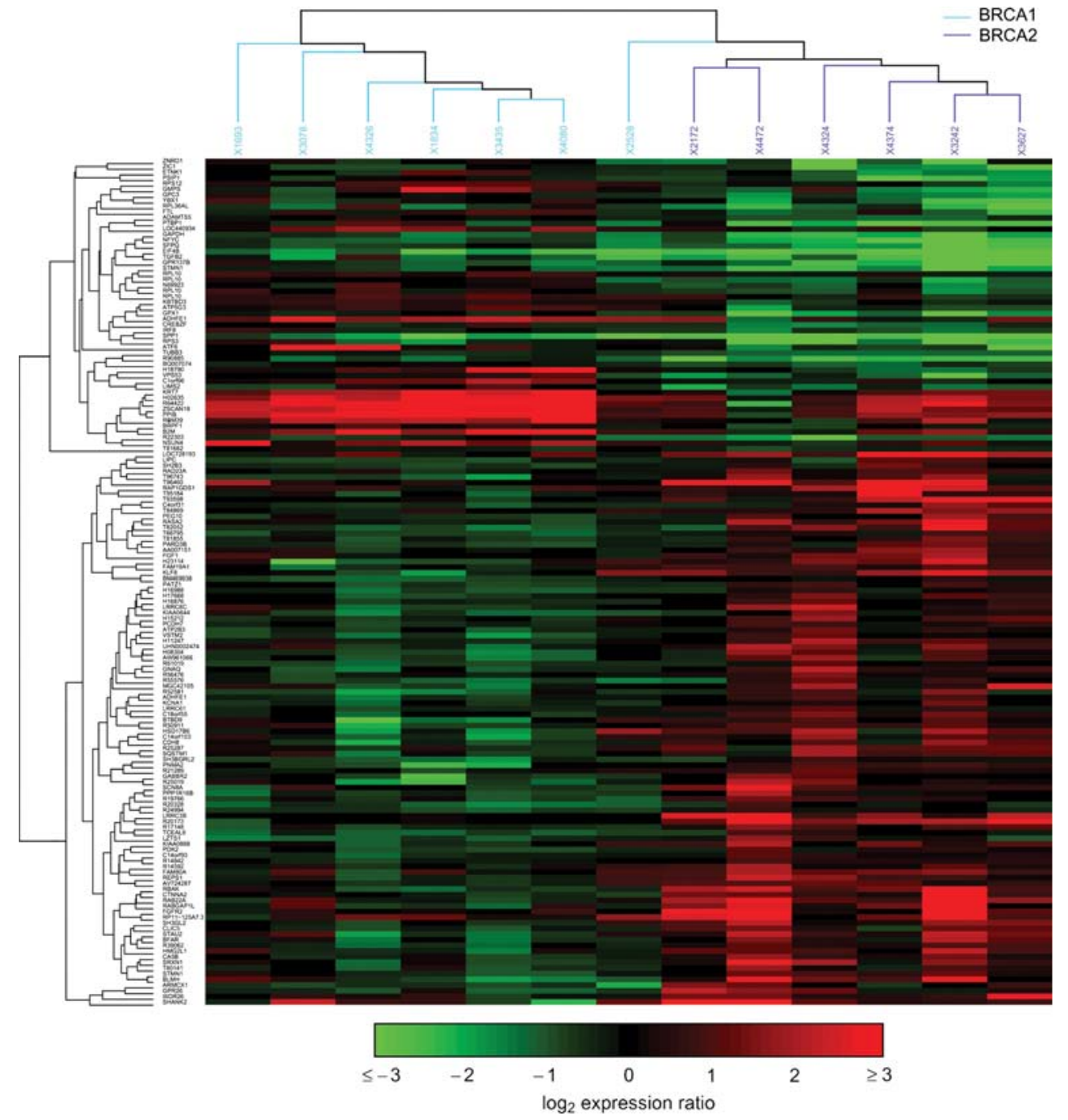


proliferation, angiogenesis, cell motility, cell adhesion, transcription and DNA repair. Involvement in the MAPK, Wnt, EGFR and TGF $\beta$ signaling pathways was identified. $B R C A 1$-associated differentially expressed genes included stathmin/oncoprotein $18 \quad(P=0.0002), \quad$ osteopontin $(P=0.002)$ and TGF $\beta 2(0.011)$.

BRCA2-associated tumors preferentially expressed 98 of the 150 clones. These genes were found to have functions related to transcription, signal transduction, cell proliferation, cell adhesion and extracellular matrix remodeling. Involvement in the MAPK signaling pathway was common. BRCA2-associated cancers were characterized by the higher relative expression of $F G F 1 \quad(P=0.003)$ and FGFR2 $(P=0.004)$.

Validation of differentially expressed genes by real time RT-PCR and IHC

To confirm the differences in gene expression, quantitative real-time RT-PCR was performed for several genes including FGF1, FGFR2, stathmin/oncoprotein 18, osteopontin and TGF $\beta 2$. Due to limited amounts of RNA, only 5 BRCA 1 and $6 B R C A 2$-associated tumor RNAs were available for RT-PCR. Similar to the microarray data, the levels of expression of osteopontin and TGF $\beta 2$ were significantly higher in the BRCAl-associated tumors compared to the BRCA2-associated tumors $(P=0.03$ and 0.001 , respectively,
Fig. 2). $B R C A 2$-associated tumors expressed higher levels of FGF1 compared to BRCA1-associated tumors and BRCAIassociated tumors had higher levels of stathmin/oncoprotein 18 compare to BRCA2-associate tumors, similar to the microarray data; but these differences however, did not reach statistical significance at the $5 \%$ level $(P=0.76$ and $P=0.28$ respectively, data not shown).

Because the levels of FGFR2 were too low to quantitate reliably in the tumors using real time RT-PCR we used immunohistochemistry (IHC) to determine the level of expression of FGFR2 in an independent set of tumors. Tissue microarrays were constructed from FFPE tumors from $64 B R C A 2$ carriers, 58 BRCAl carriers and 242 individuals without $B R C A 1$ or $B R C A 2$ mutations. A total of 19 out of $64(30 \%)$ BRCA2-associated tumors stained positive for FGFR2 compared to 3 of $50(6 \%)$ of BRCAlassociated tumors $(P=0.004$, Fig. 3$)$.

Furthermore, 25 of $151(17 \%)$ breast tumors from nonmutation carriers were positive for FGFR2, yielding statistically significant differences among the three tumor groups $(P=0.0007)$. Further analysis of all tumor groups suggested that FGFR2 expression was inversely correlated with the basal phenotype (as defined by the absence of ER, PR and HER2 expression and the presence of CK 5 or EGFR expression), with 9 of 65 (13\%) basal-like tumors staining positively for FGFR2 expression compared with 36 of $136(27 \%)$ non-basal-like breast cancers $(P=0.2)$.
Fig. 2 Scatter plots comparing the expression ratios of 6 genes obtained using both cDNA microarrays and RT-PCR in $B R C A 1$-associated and BRCA2associated tumors. The $\log 2$ expression ratios from RT-PCR (y-axis) and $\log 2$ expression ratios from cDNA microarrays (x-axis) for 6 genes from $B R C A 1$-associated (red spots) and $B R C A 2$-associated (purple spots) tumors are illustrated in the scatter plots. The relative expression ratios for both modalities for each tumor correlate positively i.e., RTPCR and expression data go in the same direction (up regulated or down regulated) for the two tumor groups
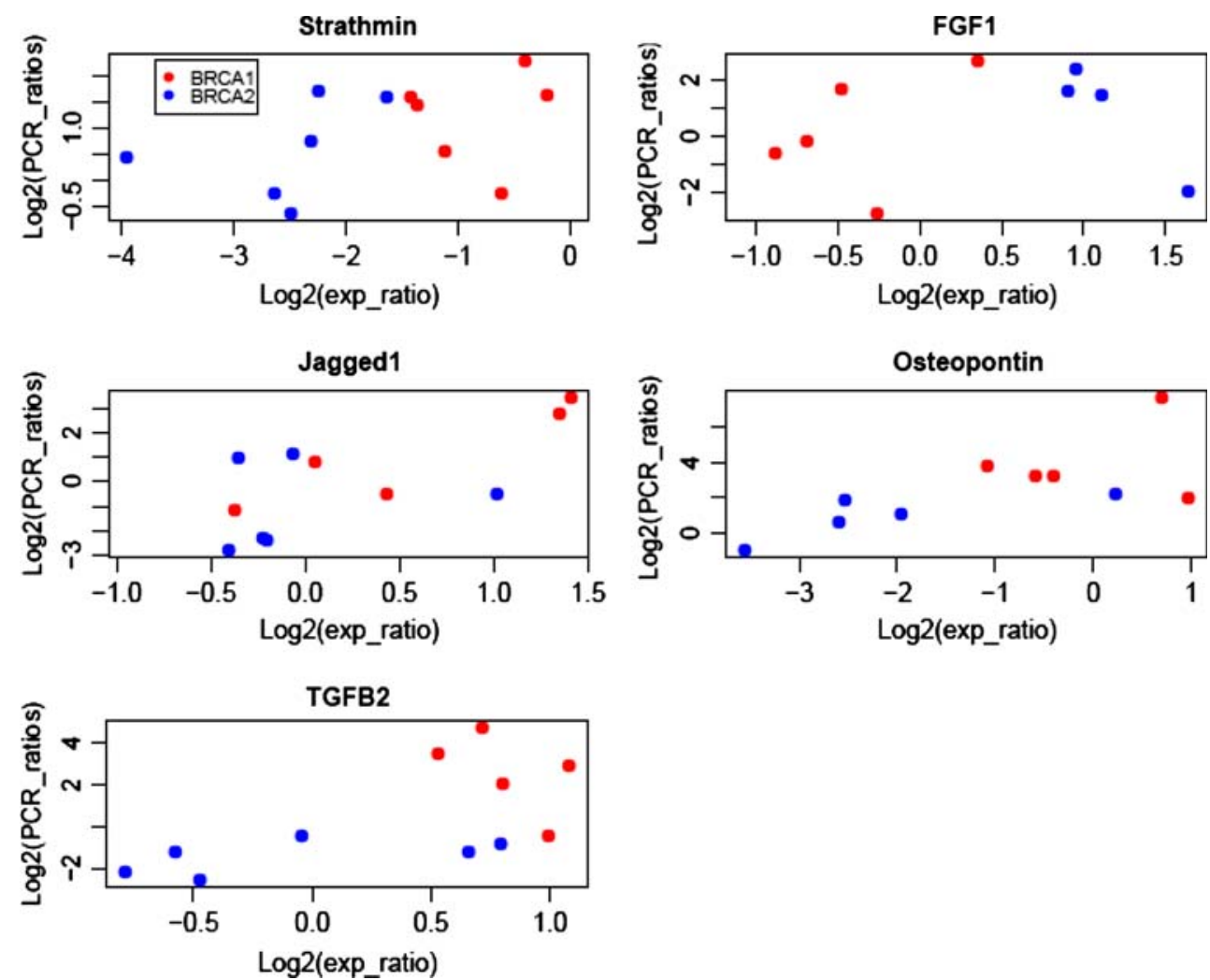
Fig. 3 Immunohistochemical and ISH studies on representative $B R C A 2$ associated and BRCA1associated tumors. Tumor (a) and $(\mathbf{b})$ are a representative $B R C A 2$-associated and BRCA1associated tumor stained for FGFR2. The cytoplasmic stain is positive in tumor (a) as evidenced by the intense brown staining and negative in tumor (b). Tumors represented in (c) and (d) are representative BRCA2-associated and BRCA1associated tumors following ISH for Jagged 1 mRNA. Tumor (c) is negative, with a vessel acting as a positive internal control whereas tumor $(\mathbf{d})$ is positive
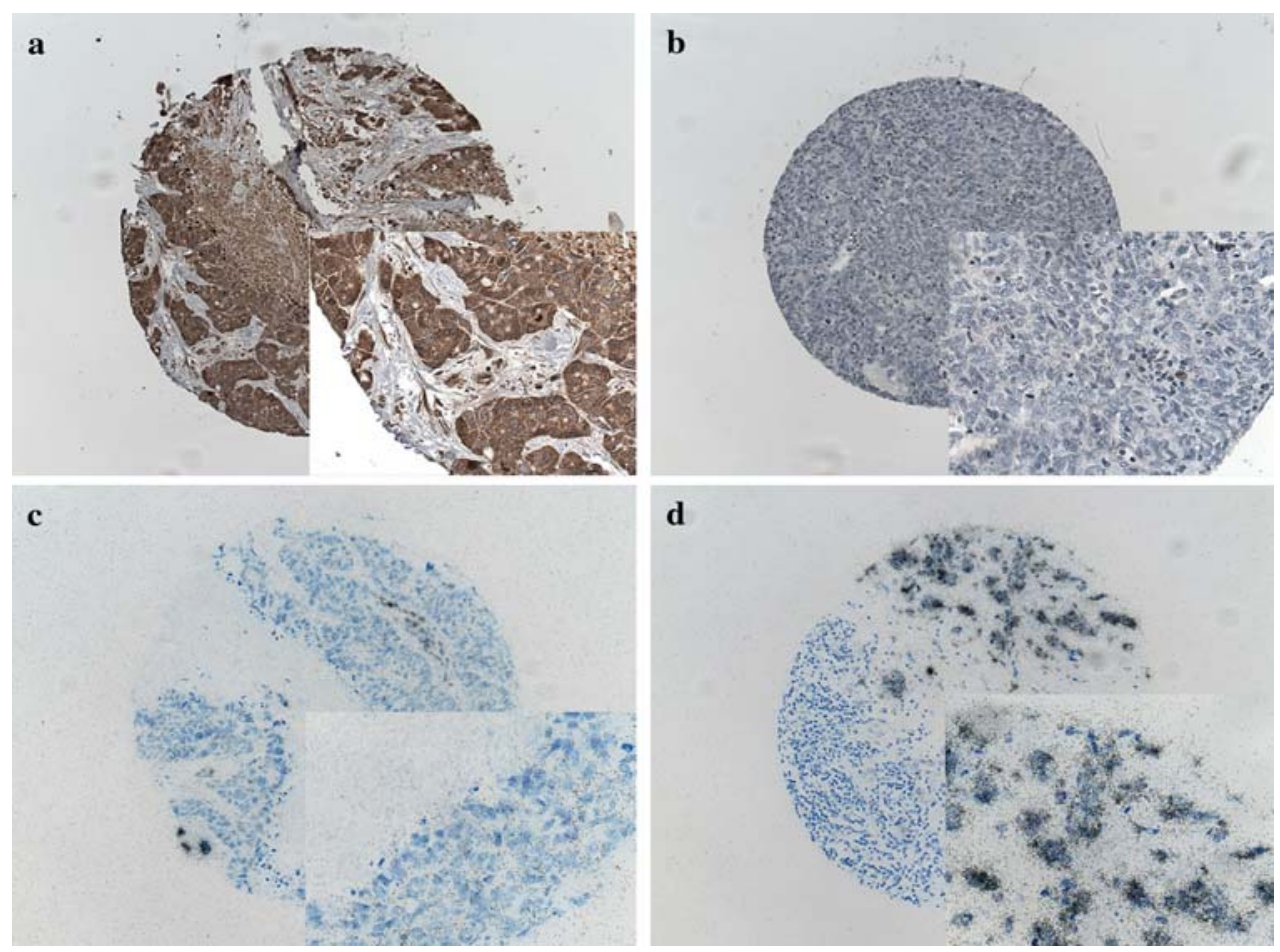

More specifically, FGFR2 negative tumors were more likely to be ER negative than FGFR2 positive tumors (49.8 vs. $26.7 \%, P=0.004$, Table 2). Similarly FGFR2 negative tumors were more likely to be negative for the luminal cytokeratin CK8/18 and p27 (14.9 vs. $2.2 \%, P=0.02$ and 36.8 vs. $13.8 \%, P=0.01$ respectively, Table 2 ) and more likely to be positive for the basal cytokeratin CK 5 and vimentin (32.0 vs. $19.2 \%, P=0.08$ and 20.3 vs. $5.9 \%$, $P=0.04$ respectively, Table 2). FGFR2 expression was also positively associated with $\mathrm{PR}$ expression $(P=0.01$, Table 2).

\section{$B R C A 1$-associated tumors}

Since $B R C A l$-associated tumors cluster with basal-like cancers $[5,6,20]$, we evaluated whether genes previously found to be more highly expressed in basal-like tumors were differentially expressed in the BRCAl compared to the $B R C A 2$ groups. Microarray based values of keratin 17, vimentin and caveolin1 tended to be higher in BRCAlassociated tumors; however, the differences in expression of these genes ( $P=0.095,0.067$, and 0.233 , respectively) did not meet our global selection cut-off.

Recently we have found that basal-like tumors tend to exhibit higher expression of Jagged 1 [21], a ligand involved in Notch pathway signaling. The Notch pathway has been shown to regulate a number of processes important in cancer including angiogenesis [22], epithelial to mesenchymal transition, and stem-cell like characteristics [23]. Using RT-PCR we found that the mean expression level of Jagged 1 tended to be elevated in BRCA1-compared to BRCA2-associated tumors (2.07 versus 0.50 , $P=0.11$ ). Because only 11 tumors were available for RTPCR, we examined the expression of Jagged 1 by mRNA ISH on the TMAs. Of 48 BRCAl-associated tumors, 17 (35\%) were positive for Jagged1 by ISH compared to 5 of 53 (9\%) BRCA2-associated cancers $(P=0.02$, Fig. 3).

\section{Discussion}

In recent years, gene expression profiling of breast cancers has improved our understanding of the heterogeneity of the disease and generated hypotheses concerning the development and progression of these cancers. Molecular signatures for BRCAl-associated tumors have been delineated, but BRCA2-associated tumors have been less well studied [5,7]. To elucidate novel genes involved in the development and progression of $B R C A 2$-associated tumors and to distinguish distinct pathways of carcinogenesis in $B R C A 1$ and BRCA2-associated tumors, we compared gene expression microarray patterns in tumors from $B R C A 1$ and BRCA2 carriers.

We found that FGFR2 and FGF1 were more highly expressed in BRCA2-associated cancers as compared to $B R C A 1$-associated breast cancers, suggesting the presence of an autocrine growth stimulatory loop. FGF1 and FGFR2 belong to a large family of ligands and receptor tyrosine kinases [24]. FGF1 is a mitogen that signals through FGFRs and subsequently activates the MAPK signaling 
Table 2 Association between FGFR2 expression and other IHC markers in all breast tumors $(n=248)$

\begin{tabular}{|c|c|c|c|c|c|}
\hline \multirow[t]{3}{*}{ Other marker } & \multicolumn{5}{|c|}{ FGFR2 } \\
\hline & \multicolumn{2}{|c|}{ Positive } & \multicolumn{2}{|c|}{ Negative } & \multirow[t]{2}{*}{$P$-value* } \\
\hline & $n^{*}$ & $\%$ & $n$ & $\%$ & \\
\hline \multicolumn{6}{|l|}{ ER } \\
\hline Positive & 33 & 73.3 & 102 & 50.3 & 0.0049 \\
\hline Negative & 12 & 26.7 & 101 & 49.8 & \\
\hline \multicolumn{6}{|l|}{ PR } \\
\hline Positive & 30 & 63.8 & 88 & 43.4 & 0.0113 \\
\hline Negative & 17 & 36.2 & 115 & 56.7 & \\
\hline \multicolumn{6}{|l|}{ Her2 } \\
\hline Positive & 5 & 10.6 & 20 & 9.9 & 0.7936 \\
\hline Negative & 42 & 89.4 & 182 & 90.1 & \\
\hline \multicolumn{6}{|l|}{ p27 } \\
\hline Positive & 25 & 86.2 & 110 & 63.2 & 0.0152 \\
\hline Negative & 4 & 13.8 & 64 & 36.8 & \\
\hline \multicolumn{6}{|l|}{ p53 } \\
\hline Positive & 13 & 27.7 & 77 & 38.9 & 0.1511 \\
\hline Negative & 34 & 72.3 & 121 & 61.1 & \\
\hline \multicolumn{6}{|l|}{ CK818 } \\
\hline Positive & 44 & 97.8 & 172 & 85.2 & 0.0207 \\
\hline Negative & 1 & 2.2 & 30 & 14.9 & \\
\hline \multicolumn{6}{|l|}{ CK5 } \\
\hline Positive & 9 & 19.2 & 65 & 32.0 & 0.0815 \\
\hline Negative & 38 & 80.9 & 138 & 68.0 & \\
\hline \multicolumn{6}{|l|}{ Vimentin } \\
\hline Positive & 2 & 5.9 & 36 & 20.3 & 0.0445 \\
\hline Negative & 32 & 94.1 & 141 & 79.7 & \\
\hline
\end{tabular}

cascade. Overexpression of FGF1 in breast cancer cell lines has been shown to result in increased anchorage independent growth and reduced requirement for estrogen in vitro and to increased tumorigenicity, angiogenesis and metastatic behaviour in vivo $[25,26] . F G F 1$ is located at $5 \mathrm{q} 31$ a region of the genome often subject to loss in BRCAIassociated cancers [27] and basal-like cancers [28].

FGFR2, located at 10q26, encodes at least two receptor isoforms FGFR2-IIIb and FGFR2-IIIc. FGFR2-IIIc is mainly expressed in tissues of mesenchymal origin, whereas FGFR2IIIb is expressed in the epithelium of many organs including the mammary gland $[29,30]$. The FGFRs in general and FGFR2 specifically have roles in embryogenesis, development and carcinogenesis. FGFR2 mRNA is expressed in many carcinoma cell lines [31-33] and in the breast cancer cell lines examined, FGFR2 expression contributes to their invasive phenotype. Similarly, overexpression of the receptor in normal human mammary epithelial (HME) cells leads to their transformation [34]. These properties are attributable to the activation of the mitogen-activated protein kinase (MAPK) and phosphatidylinositol 3-kinase (PI3K) signaling cascades [34]. Moreover, the gene locus (10q26) is amplified in 2-10\% of breast cancers and its expression has been reported in 50 $100 \%$ of human breast cancers, with high-level expression confined to $4 \%-12 \%$ of cases $[35,36]$. It has also been associated with estrogen (ER) and progesterone (PR) receptor expression and improved overall and disease free survival [35]. Moreover in two recent independent genome wide association studies (GWAS) single nucleotide polymorphisms (SNPs) within intron 2 of FGFR2 have been causally associated with increased risk of both familial and sporadic breast cancer [37, 38]. Furthermore this susceptibility locus was associated with younger age of onset and bilateral disease [37]. The authors speculate that the SNPs have functional effects and that the association with breast cancer risk is mediated through regulation of FGFR2 expression possibly through interaction with the ER. In our study we have shown that FGFR2 expression is positively associated with the $B R C A 2$ genotype, a tumor group that we have previously shown to be predominantly ER-positive luminal-type tumors [1]. In addition, we found that FGFR2 expression was positively associated with PR expression, a weak prognostic and predictive factor in breast cancer and often regarded as indicative of a functional ER pathway [39]. Conversely, FGFR2 expression was negatively correlated with basal-like breast cancers which are known to be ER negative and to carry an adverse prognosis $[5,6]$.

Our expression studies support previous reports indicating that $B R C A l$-associated breast cancers have a basallike profile $[3,5]$. In the $B R C A l$-associated tumor group, we detected elevated expression of a number of genes, including keratin 17 [5, 6], vimentin, and caveolin 1 [20], that have previously been linked to the basal-like cancers. Our results also suggest that Y-box binding protein-1 is more highly expressed in $B R C A l$-associated breast cancers $(P=0.001)$. This protein has recently been demonstrated to transcriptionally induce EGFR which is commonly overexpressed in basal-like cancers [40]. In addition we found that stathmin/oncoprotein 18 and osteopontin were overexpressed in BRCAl-associated tumors relative to $B R C A 2$-associated tumors. The gene for stathmin, located at $1 \mathrm{p} 36$, is one of the 70 genes that compose the ' 70 gene classifier' that predicts poor prognosis in sporadic breast cancer [41] a classifier that correlates closely with the poor performing subgroups from the intrinsic gene set of which basal-like cancers are one [42]. Stathmin is a microtubule depolymerizing protein involved in cell cycle progression and cell motility. It is highly expressed in a number of human malignancies, including breast cancers where it is negatively correlated with ER expression and positively correlated with grade, aneuploidy, proliferation and mutant 
p53 [43], all characteristic features of BRCAl-associated and basal-like cancers $[3,5,6]$.

Osteopontin, a secreted phosphoprotein, has been shown to interact with a diverse range of factors including integrins, CD44, TGF $\alpha$, EGFR, Met and VEGF leading to the enhancement of cellular migration, invasion, survival and angiogenesis. [44-46]. Osteopontin is expressed in a wide variety of human malignancies and its expression has been reported to be correlated with poor prognosis in breast cancer [47].

Novel results from our expression study include the identification of members of the Notch and TGF $\beta$ signaling pathways, Jagged 1 and TGF $\beta 2$ respectively, as being more highly expressed in BRCAl-associated tumors. Both of these pathways are highly conserved through evolution and play important roles in development, differentiation and tumorigenesis [48, 49]. Moreover, both pathways are known to play a role in mammary stem cell maintenance or renewal $[50,51]$. Dontu et al. have shown that Notch signaling can act on mammary stem cells to promote selfrenewal, on early progenitor cells to promote proliferation, and on multipotent progenitor cells, facilitating myoepithelial cell lineage specific commitment and proliferation [50]. Furthermore, patients whose breast cancers express Jagged 1 have been found to have a poorer prognosis [17]. Shipitsin and colleagues showed that TGF $\beta$ signaling is upregulated in normal breast stem cells and their malignant counterparts and has prognostic effects [51].

In conclusion, our molecular profiling demonstrates that $B R C A 1$-associated and BRCA2-associated tumors have distinct molecular profiles. Our results confirm the known link between BRCAl-associated tumors and the basal-like signature and highlight a link between the Notch and TGF $\beta$ pathways and BRCAl-associated tumorigenesis. In contrast the data suggest that BRCA2-associated tumors express higher levels of FGFR2 and FGF1, suggesting the existence of an autocrine loop leading to downstream pleiotrophic cellular effects.

Acknowledgements The authors thank Dr. Brendan Dickson and Hui Zhang for their technical assistance. Grant numbers and sources of support: This work was supported by the Canadian Breast Cancer Foundation and the National Cancer Institute under RFA \#CA-95-003 as part of the Breast Cancer Family Registry (BreastCFR).

Open Access This article is distributed under the terms of the Creative Commons Attribution Noncommercial License which permits any noncommercial use, distribution, and reproduction in any medium, provided the original author(s) and source are credited.

\section{References}

1. Bane AL, Beck JC et al (2007) BRCA2 mutation-associated breast cancers exhibit a distinguishing phenotype based on morphology and molecular profiles from tissue microarrays.
Am J Surg Pathol 31:121-128. doi:10.1097/01.pas.0000213351. 49767.0f

2. Lakhani SR, Jacquemier J et al (1998) Multifactorial analysis of differences between sporadic breast cancers and cancers involving BRCA1 and BRCA2 mutations. J Natl Cancer Inst 90:11381145. doi:10.1093/jnci/90.15.1138

3. Lakhani SR, Reis-Filho JS et al (2005) Prediction of BRCA1 status in patients with breast cancer using estrogen receptor and basal phenotype. Clin Cancer Res 11:5175-5180. doi:10.1158/ 1078-0432.CCR-04-2424

4. Palacios J, Honrado E et al (2005) Phenotypic characterization of BRCA1 and BRCA2 tumors based in a tissue microarray study with 37 immunohistochemical markers. Breast Cancer Res Treat 90:5-14. doi:10.1007/s10549-004-1536-0

5. Sorlie T, Tibshirani R et al (2003) Repeated observation of breast tumor subtypes in independent gene expression data sets. Proc Natl Acad Sci USA 100:8418-8423. doi:10.1073/pnas.0932692100

6. Nielsen TO, Hsu FD et al (2004) Immunohistochemical and clinical characterization of the basal-like subtype of invasive breast carcinoma. Clin Cancer Res 10:5367-5374. doi:10.1158/ 1078-0432.CCR-04-0220

7. Hedenfalk I, Duggan D et al (2001) Gene-expression profiles in hereditary breast cancer. N Engl J Med 344:539-548. doi: 10.1056/NEJM200102223440801

8. John EM, Hopper JL et al (2004) The breast cancer family registry: an infrastructure for cooperative multinational, interdisciplinary and translational studies of the genetic epidemiology of breast cancer. Breast Cancer Res 6:R375-R389. doi:10.1186/bcr801

9. Sutherland HJ, Lacroix J et al (2001) The cooperative familial registry for breast cancer studies: design and first year recruitment rates in Ontario. J Clin Epidemiol 54:93-98. doi:10.1016/ S0895-4356(00)00263-8

10. Andrulis IL, Anton-Culver $\mathrm{H}$ et al (2002) Comparison of DNAand RNA-based methods for detection of truncating BRCA1 mutations. Hum Mutat 20:65-73. doi:10.1002/humu.10097

11. Tavassoli T, Devilee P (2003) Pathology \& Genetics. Tumours of the breast and female genital organs. IARC Press, Lyon

12. Elston CW, Ellis IO (1991) Pathological prognostic factors in breast cancer. I. The value of histological grade in breast cancer: experience from a large study with long-term follow-up. Histopathology 19:403-410. doi:10.1111/j.1365-2559.1991.tb00229.x

13. Perou CM, Sorlie T et al (2000) Molecular portraits of human breast tumours. Nature 406:747-752. doi:10.1038/35021093

14. Simon R, Korn E (2003) Design and analysis of DNA microarray investigations. Springer-Verlag, New York

15. Yang YH, Dudoit $\mathrm{S}$ et al (2002) Normalization for cDNA microarray data: a robust composite method addressing single and multiple slide systematic variation. Nucl Acids Res 30:e15. doi:10.1093/nar/30.4.e15

16. Smyth GK (2005) Limma: linear models for microarray data. In: Bioinformatic and computional biology solutions using $\mathrm{R}$ and bioconductor. Springer, New York

17. Reedijk M, Odorcic S et al (2005) High-level coexpression of JAG1 and NOTCH1 is observed in human breast cancer and is associated with poor overall survival. Cancer Res 65:8530-8537. doi:10.1158/0008-5472.CAN-05-1069

18. Allred DC, Harvey JM et al (1998) Prognostic and predictive factors in breast cancer by immunohistochemical analysis. Mod Pathol 11:155-168

19. Tusher VG, Tibshirani R et al (2001) Significance analysis of microarrays applied to the ionizing radiation response. Proc Natl Acad Sci USA 98:5116-5121. doi:10.1073/pnas.091062498

20. Pinilla SM, Honrado E et al (2006) Caveolin-1 expression is associated with a basal-like phenotype in sporadic and hereditary breast cancer. Breast Cancer Res Treat 99:85-90. doi:10.1007/ s10549-006-9184-1 
21. Reedijk M, Pinnaduwage D et al (2007) JAG1 expression is associated with a basal phenotype and recurrence in lymph nodenegative breast cancer. Breast Cancer Res Treat, Nov 8 [Epub]

22. Uyttendaele H, Ho J et al (2001) Vascular patterning defects associated with expression of activated Notch4 in embryonic endothelium. Proc Natl Acad Sci USA 98:5643-5648. doi: 10.1073/pnas.091584598

23. Varnum-Finney B, Purton LE et al (1998) The notch ligand, jagged-1, influences the development of primitive hematopoietic precursor cells. Blood 91:4084-4091

24. Dailey L, Ambrosetti D et al (2005) Mechanisms underlying differential responses to FGF signaling. Cytokine Growth Factor Rev 16:233-247. doi:10.1016/j.cytogfr.2005.01.007

25. McLeskey SW, Ding IY et al (1994) MDA-MB-134 breast carcinoma cells overexpress fibroblast growth factor (FGF) receptors and are growth-inhibited by FGF ligands. Cancer Res 54:523-530

26. Zhang L, Kharbanda S et al (1997) MCF-7 breast carcinoma cells overexpressing FGF-1 form vascularized, metastatic tumors in ovariectomized or tamoxifen-treated nude mice. Oncogene 15:2093-2108. doi:10.1038/sj.onc. 1201386

27. van Beers EH, van Welsem T et al (2005) Comparative genomic hybridization profiles in human BRCA1 and BRCA2 breast tumors highlight differential sets of genomic aberrations. Cancer Res 65:822-827

28. Bergamaschi A, Kim YH et al (2006) Distinct patterns of DNA copy number alteration are associated with different clinicopathological features and gene-expression subtypes of breast cancer. Genes Chromosomes Cancer 45:1033-1040. doi:10.1002/ gcc. 20366

29. De Moerlooze L, Spencer-Dene B et al (2000) An important role for the IIIb isoform of fibroblast growth factor receptor 2 (FGFR2) in mesenchymal-epithelial signalling during mouse organogenesis. Development 127:483-492

30. Mailleux AA, Spencer-Dene B et al (2002) Role of FGF10/ FGFR2b signaling during mammary gland development in the mouse embryo. Development 129:53-60

31. Bansal GS, Cox HC et al (1997) Expression of keratinocyte growth factor and its receptor in human breast cancer. $\mathrm{Br} \mathrm{J}$ Cancer 75:1567-1574

32. Carstens RP, Eaton JV et al (1997) Alternative splicing of fibroblast growth factor receptor 2 (FGF-R2) in human prostate cancer. Oncogene 15:3059-3065. doi:10.1038/sj.onc.1201498

33. Otte JM, Schmitz F et al (2000) Expression of keratinocyte growth factor and its receptor in colorectal cancer. Eur J Clin Invest 30:222-229. doi:10.1046/j.1365-2362.2000.00617.x

34. Moffa AB, Tannheimer SL et al (2004) Transforming potential of alternatively spliced variants of fibroblast growth factor receptor 2 in human mammary epithelial cells. Mol Cancer Res 2:643-652

35. Blanckaert VD, Hebbar M et al (1998) Basic fibroblast growth factor receptors and their prognostic value in human breast cancer. Clin Cancer Res 4:2939-2947

36. Penault-Llorca F, Bertucci F et al (1995) Expression of FGF and FGF receptor genes in human breast cancer. Int J Cancer 61:170176. doi:10.1002/ijc.2910610205
37. Easton DF, Pooley KA et al (2007) Genome-wide association study identifies novel breast cancer susceptibility loci. Nature 447(7148):1087-1093

38. Hunter DJ, Kraft P et al (2007) A genome-wide association study identifies alleles in FGFR2 associated with risk of sporadic postmenopausal breast cancer. Nat Genet 39:870-874. doi: $10.1038 / \mathrm{ng} 2075$

39. Fitzgibbons PL, Page DL et al (2000) Prognostic factors in breast cancer. College of American pathologists consensus statement 1999. Arch Pathol Lab Med 124:966-978

40. Stratford AL, Habibi G et al (2007) Epidermal growth factor receptor (EGFR) is transcriptionally induced by the Y-box binding protein-1 (YB-1) and can be inhibited with Iressa in basal-like breast cancer providing a potential target for therapy. Breast Cancer Res 9:R61. doi:10.1186/bcr1767

41. van de Vijver MJ, He YD et al (2002) A gene-expression signature as a predictor of survival in breast cancer. N Engl J Med 347:1999-2009. doi:10.1056/NEJMoa021967

42. Fan C, Oh DS et al (2006) Concordance among gene-expressionbased predictors for breast cancer. N Engl J Med 355:560-569. doi:10.1056/NEJMoa052933

43. Brattsand G (2000) Correlation of oncoprotein 18/stathmin expression in human breast cancer with established prognostic factors. Br J Cancer 83:311-318. doi:10.1054/bjoc.2000.1264

44. Shijubo N, Uede $T$ et al (2000) Vascular endothelial growth factor and osteopontin in tumor biology. Crit Rev Oncog 11:135146

45. Tuck AB, Elliott BE et al (2000) Osteopontin-induced, integrindependent migration of human mammary epithelial cells involves activation of the hepatocyte growth factor receptor (met). J Cell Biochem 78:465-475. doi:10.1002/1097-4644(20000901)78:3< 465::AID-JCB $11>3.0 . \mathrm{CO} ; 2-\mathrm{C}$

46. Tuck AB, Hota $C$ et al (2003) Osteopontin-induced migration of human mammary epithelial cells involves activation of EGF receptor and multiple signal transduction pathways. Oncogene 22:1198-1205. doi:10.1038/sj.onc.1206209

47. Allan AL, George R et al (2006) Role of the integrin-binding protein osteopontin in lymphatic metastasis of breast cancer. Am J Pathol 169:233-246. doi:10.2353/ajpath.2006.051152

48. Moses HL, Serra R (1996) Regulation of differentiation by TGFbeta. Curr Opin Genet Dev 6:581-586. doi:10.1016/S0959437X(96)80087-6

49. Roberts AB, Wakefield LM (2003) The two faces of transforming growth factor beta in carcinogenesis. Proc Natl Acad Sci USA 100:8621-8623. doi:10.1073/pnas.1633291100

50. Dontu G, Jackson KW et al (2004) Role of notch signaling in cell-fate determination of human mammary stem/progenitor cells. Breast Cancer Res 6:R605-R615. doi:10.1186/bcr920

51. Shipitsin M, Campbell LL et al (2007) Molecular definition of breast tumor heterogeneity. Cancer Cell 11:259-273. doi:10.1016/ j.ccr.2007.01.013 